\title{
Análisis multivariado a los factores relacionados con el aprendizaje móvil en la educación superior en Colombia ${ }^{1}$
}

\author{
Multivariate Analysis of Elements Related to Mobile Learning in Higher Education in Colombia ${ }^{2}$
}

\section{Análise multivariada de fatores relacionados à aprendizagem móvel no ensino superior na Colômbia ${ }^{3}$}

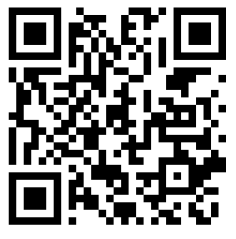

Recibido • Received • Recebido: 03 / 10 / 2016

Corregido • Revised • Revisado: 17 / 04 / 2018

Aceptado • Accepted • Aprovado: 07/ 06 / 2018

\footnotetext{
${ }^{1}$ Este artículo muestra y amplía una parte de los resultados encontrados en la tesis de maestría Factores que contribuyen y dificultan el desarrollo de la enseñanza aprendizaje mediada por dispositivos móviles en instituciones de educación superior en Colombia (Estrada-Villa, 2014).

${ }^{2}$ This article shows and extends a part of the results found in the master's degree thesis titled Factors that contribute to and hinder the development of mobile-mediated teaching learning in higher education institutions in Colombia (Estrada-Villa, 2014).

${ }^{3}$ Este artigo demonstra e amplia uma parte dos resultados da sua teses do mestrado Fatores que contribuem $e$ dificultam o desenvolvimento do ensino-aprendizagem através dos dispositivos moveis nas instituições de educação superior na Colômbia (Estrada-Villa, 2014).

${ }^{4}$ Magíster en Informática Educativa de la Universidad de La Sabana. Profesora Investigadora Escuela de Postgrados Fuerza Aérea Colombiana - Grupo CIPAER.

${ }^{5}$ Doctor en Ciencias de la Educación. Profesor e investigador asociado Maestría en Informática Educativa, Centro de Tecnologías para la Academia Universidad de La Sabana.
} 
doi: http://dx.doi.org/10.15359/ree.22-3.6

URL: http://www.una.ac.cr/educare

CORREO: educare@una.cr

Resumen: El presente artículo tiene como objetivo identificar, a través de un análisis de componentes principales, los factores relacionados con el aprendizaje móvil o $m$-learning en educación superior. Para esto, se llevó a cabo una investigación mixta, que retomó los hallazgos de una investigación más amplia, que tuvo como resultado la determinación de catorce factores, y que este artículo reduce a tres a través de un análisis multifactorial. Además, para la recolección de los datos se aplicó un instrumento con escala tipo Likert a 30 personas expertas investigadoras universitarias. Para el procesamiento de los datos se empleó un análisis de componentes principales, que arrojó como resultado los siguientes factores: capacitación, características de estudiantes y falta de gestión administrativa, los cuales guardan correlación con los factores falta de lineamientos m-learning y el de infraestructura. En conclusion, los factores extraídos deben ser tenidos en cuenta a la hora de trazar políticas y lineamientos para incluir el aprendizaje móvil en la educación superior, así como en los planes de formación docente, en respuesta a lo que el estudiantado está demandando.

Palabras claves: Aprendizaje móvil; enseñanza-aprendizaje; educación superior; TIC.

Abstract: This article shows the elements related to mobile learning in higher education; a multivariate analysis (the main focus of this article) on these elements was performed. For this, mixed research was conducted that took part of the results obtained on a previous research project. In these results, fourteen elements were identified; now, this article reduces them to three through a multifactorial analysis. Besides, to collect the data, a Likert-type instrument was applied to 30 university researchers. To process the data a multivariate analysis was implemented, resulting in the following elements: the training element and the student's characteristics; the lack of management that correlates with the lack of m-learning guidelines and lack of infrastructure. In conclusion, the elements extracted should be taken into account when drawing guidelines to include mobile learning in higher education, as well as teacher training plans, in response to what students require.

Keywords: Mobile learning; teaching-learning; higher education; ICT.

Resumo: O objetivo deste artigo é identificar, através de uma análise de componentes principais, os fatores relacionados à aprendizagem móvel ou m-learning no ensino superior. Para isso, realizouse uma investigação mista, que retomou as descobertas de uma investigação mais ampla, que resultou na determinação de quatorze fatores, e que este artigo reduz a três, por meio de uma análise multifatorial. Ademais, para a coleta dos dados, aplicou-se um instrumento com escala tipo Likert a 30 pesquisadores universitários especialistas na matéria. Para o processamento dos dados, empregou-se uma análise de componentes principais, que teve como resultado os seguintes fatores: capacitação, características de estudantes e falta de gerenciamento administrativo, os quais guardam correlação com os fatores de falta de lineamentos m-learning e de infraestrutura. Em conclusão, os fatores extraídos devem ser levar em conta à hora de traçar políticas e lineamentos para incluir a aprendizagem móvel no ensino superior, bem como nos planejamentos de formação docente, como resposta as demandas estudantis.

Palavras-chave: Aprendizagem móvel; ensino-aprendizagem; Educação superior; TIC 


\section{Introducción}

La presente investigación se basa en el aprendizaje móvil, mejor conocido como m-learning; O'Malley et al. (2005) lo presentan como un campo de la educación que constituye actualmente un contexto creciente de interés, donde se pretende, fundamentalmente, aplicar técnicas de aprendizaje basadas en la tecnología de los dispositivos móviles (Attewell, 2005). A su vez, Quinn (2000, citado en Sánchez, Sáenz, Muñoz, Ramírez y Martín, 2009) define la computación móvil en los siguientes términos:

Una computación portátil con alta interactividad, conectividad total y que permite un alto nivel de procesamiento. Junto con esto agrega que considera un dispositivo pequeño que está siempre en red, que permite una fácil entrada de datos a través de un lápiz específico, voz o por un teclado si es necesario, y la habilidad de ver imágenes con alta resolución y alta calidad de sonido. (p. 2)

El uso de dispositivos va en aumento (Attewell, 2005) y ha sido una tendencia de gran crecimiento en los últimos años. Países como Korea han estado trabajando modelos de aprendizaje ubicuo a través del uso de dispositivos móviles, para desarrollar procesos de alfabetización digital. Lo anterior demuestra grandes avances en el diseño de propuestas en este aspecto (Estrada-Villa, 2016), porque transmite una visión de la educación que conecta a través de diferentes estancias, dado que el aprendizaje no se produce solo en el aula, sino también en el hogar, en el parque, en el museo o la biblioteca, y se da a través de la interacción con el ambiente (Bruce, 2008).

En concordancia, a nivel mundial, el Informe Horizon (Johnson, Adams y Cummins, 2012) propuso abrir a la educación distintas herramientas vinculadas con el aprendizaje móvil, y fue enfático en mencionar que en un plazo de un año se integrarían las aplicaciones (APP) y las tabletas; que en un periodo de tres años arribaría el aprendizaje basado en juegos y que en uno de cinco años se tendría el internet de las cosas.

\section{Antecedentes}

En la revisión de la bibliografía se han encontrado varias experiencias que vinculan el aprendizaje móvil con actividades de enseñanza-aprendizaje en educación superior. Dicho aprendizaje es entendido por la Unesco (2012), en su publicación Mobile Learning and Policies. Key issues to consider, como la educación que implica el uso de dispositivos móviles para permitir el aprendizaje en cualquier momento y en cualquier lugar. A partir de lo anterior y para documentación practica y profundización del tema m-learning, se realizó la búsqueda y el levantamiento de experiencias de aprendizaje móvil en educación superior, las cuales se presentan a continuación. 
doi: http://dx.doi.org/10.15359/ree.22-3.6

URL: http://www.una.ac.cr/educare

CORREO: educare@una.cr

e-sanitas: ofrece una amplia gama de soluciones educativas bajo demanda, para el desarrollo profesional continuo por medio de las nuevas tecnologías de información y comunicación (e-learning,b-learning,m-learning). Actualmente,e-sanitas permitelacombinación única de la educación virtual, mediante un equipo multidisciplinario y versátil que elimina los mayores obstáculos de la tecnología a través del enfoque al público usuario final y la gestión del conocimiento. e-sanitas hace esto posible gracias a varias fuerzas confluentes: alineamiento de estrategias entre el objetivo y el contenido, pedagogía dirigida al público usuario, arquitectura de contenido, entre otras, las cuales integran el aprendizaje móvil (Estrada-Villa, 2014).

Bluegenesis: es una plataforma académica que utiliza los teléfonos móviles para apoyar la comunicación y el proceso de enseñanza-aprendizaje a nivel universitario. Mediante el uso de la conectividad bluetooth, el profesorado puede enviar desde sus portátiles preguntas, pruebas, contenidos y mensajes a teléfonos móviles del estudiantado. Este último puede leer los materiales, responder preguntas y recibir comentarios en sus teléfonos móviles (Estrada-Villa, 2014).

SENA Móvil: facilita acceder el material educativo de un curso en formatos de texto, audio y video, desde teléfonos celulares. El sistema permite desarrollar y enviar actividades de formación al personal tutor. Esta modalidad hace parte de la oferta de formación que se soporta mediante el m-learning, con contenidos pedagógicos que se pueden desarrollar con la mencionada metodología. De esta manera, el Servicio Nacional de Aprendizaje (SENA) provee a la población colombiana nuevas posibilidades tecnológicas cada vez más flexibles, para eliminar barreras y permitir el acceso a la formación profesional que se imparte a través de ambientes virtuales de aprendizaje (Estrada-Villa, 2014).

Experiencia de podcasting en la enseñanza de una segunda lengua: la finalidad de esta experiencia es mejorar las habilidades auditivas apoyadas con aprendizaje móvil. Para ello, se implementaron archivos de audio llamados podcasts a través de dispositivos móviles. Se diseñaron siete podcasts teniendo en cuenta las necesidades y errores estudiantiles en el aprendizaje de un idioma. Los resultados de esta experiencia fueron favorables porque el estudiantado es consciente de la falencia que posee y su deseo de mejorarla (Estrada-Villa, 2014).

Uniminuto Teach me: el Tablet PC es una herramienta móvil para incentivar el aprendizaje de las matemáticas; por tanto, estos dispositivos se convirtieron en el insumo para el desarrollo del proyecto investigativo institucional Teach me (Technology, Engineering And Calculus Hewlett-Packard [HP] Mobile Environment), que involucra la inclusión de esta tecnología en tres de los cursos de matemáticas que presentaban mayor pérdida académica en la Facultad de Ingeniería de la Uniminuto. Finalmente, los resultados de la experiencia se presentaron desde tres aspectos: el trabajo de estudiantes, la interacción didáctica y los aportes de la herramienta móvil al aprendizaje de la función lineal (Estrada-Villa, 2014). 
EAFIT didáctica del cálculo: el objetivo general de la investigación fue describir a partir de un estudio de caso la calidad del aprendizaje que evidencian estudiantes de las carreras de Ingeniería de la Universidad EAFIT de la asignatura de Cálculo cuando se implementa una propuesta didáctica con tecnología móvil (Pocket PC). Los resultados arrojaron que cuando se emplea de manera didáctica la tecnología móvil, se desarrollan habilidades cognitivas, metacognitivas, tecnológicas y colaborativas que le permiten al estudiantado desarrollar competencias para la resolución de problemas (Estrada -Villa, 2014).

Hasta aquí, se presentó un breve estado del arte de la exploración inicial sobre algunas experiencias de aprendizaje móvil en educación superior.

\section{Método}

Este trabajo es una combinación de dos tipos de investigación que, según Hernández, Fernández y Baptista (2010) se clasifica en una investigación mixta, porque desde lo cuantitativo contrastólos datosobtenidos con una perspectiva clara a partir del análisis estadístico multivariado, con el fin de garantizar la pertinencia de los factores investigados, y desde lo cualitativo buscó la interpretación de los factores, lo que permitió hacer inferencias de los datos obtenidos. Por lo tanto, el método mixto es complementario y da una visión estadística y descriptiva de los factores que están relacionados con el aprendizaje móvil en educación superior.

El presente artículo forma parte de una investigación más amplia denominada Factores que contribuyen y dificultan el desarrollo de la enseñanza aprendizaje mediada por dispositivos móviles en instituciones de educación superior en Colombia, realizada por Estrada-Villa (2014) entre 2012 y 2014. En esta se aplicó el instrumento denominado "Cuestionario Az Móvil Colombia" a 176 docentes, que orientan sus clases en pregradoy posgrado en diferentes instituciones de educación superior (IES) en Colombia. Los resultados fueron analizados en Atlas.ti; de la categorización en el análisis cualitativo emergieron catorce factores relacionados con aprendizaje móvil (ver tabla 1).

Tabla 1: Factores relacionados con el m-learning

\begin{tabular}{ll}
\multicolumn{1}{c}{ Factores que posibilitan m-learning IES } & \multicolumn{1}{c}{ Factores que dificultan m-learning IES } \\
\hline * Factor infraestructura & * Actitud y desinterés \\
* Factor capacitación y formación docente & ${ }^{*}$ Falta capacitación \\
* Factor políticas IES & $*$ Falta lineamientos m-learning y políticas IES \\
* Factor actitud e interés docente & * Falta infraestructura \\
* Factor características del estudiantado & * Falta gestión administrativa \\
* Factor conocimiento y habilidades docentes & * Desconocimiento \\
* Factor gestión administrativa & \\
* Factor área de conocimiento y campo de estudio & \\
\hline
\end{tabular}

Nota: Tomado de Estrada-Villa (2014, pp. 86, 92). 
doi: http://dx.doi.org/10.15359/ree.22-3.6

URL: http://www.una.ac.cr/educare

CORREO: educare@una.cr

En el presente artículo, los factores relacionados en la Tabla 1 son reducidos mediante un análisis multivariado; esto, con el fin de que se pierda la menor cantidad de información posible y que quedaran aquellos factores que posiblemente se puedan intervenir en la gestión de las universidades colombianas interesadas en incluir los dispositivos móviles.

Para ello, se seleccionó el análisis de componentes principales (ACP) y ANOVA Multifactorial, técnicas estadísticas de síntesis de la información o reducción del número de variables. Un aspecto fundamental en la estadística de los métodos multivariados, según Terrádez (s. f. p. 1), "es la interpretación de los factores, ya que ésta no viene dada a priori, sino que [se deduce] tras observar la relación de los factores con las variables", a partir de la confianza y la varianza de los datos, de tal manera que se pudiese validar la correlación entre estos.

\section{Instrumento}

Dentro del desarrollo de la investigación y para la validación de los factores relacionados con el m-learning en las IES en Colombia, se diseñó la "Encuesta Factores Az Móvil"con respuesta tipo escala Likert, donde cinco es el valor más alto y uno el más bajo. El instrumento se validó mediante tablas de frecuencias, con valores de respuestas del $87 \%$, y se implementó a través de un formulario en Google Drive Inc, desde diciembre de 2013 hasta febrero de 2014.

\section{Población y muestra}

La muestra la constituyeron 30 personas expertas en TIC e investigadoras de diferentes universidades del país (Estrada-Villa, 2014); los datos se recogieron mediante un muestreo no probabilístico tipo "bola de nieve" (Bernal, 2000), el cual inició con el personal docente y personas investigadoras del Centro de Tecnologías para la Academia de la Universidad de la Sabana, donde se buscó a personal experto investigador en temas relacionados con tecnología educativa, principalmente con el m-learning.

\section{Resultados y análisis}

Para la recogida de los datos se empleó el instrumento, descrito anteriormente, y para el análisis de los datos se trabajó con ACP y ANOVA multifactorial, donde se cruzaron y analizaron los datos de la muestra utilizando el software estadístico Statgraphics, de tal manera que se pudieran validar la correlación entre los diferentes factores.

A continuación, se presentan los resultados de acuerdo con cada análisis realizado. Primero se relacionan la información de los factores que favorecen y segundo de los factores que dificultan. 


\section{Análisis multifactorial. Factores quefavorecen el aprendizaje móvil en educación superior}

Para iniciar, en la Figura 1 se presentan los resultados arrojados por la encuesta tipo Likert, donde se observan los porcentajes en las respuestas que dio el equipo docente investigador a los factores que favorecen la inclusión del aprendizaje móvil en la educación superior. El equipo, en un $83 \%$, está de acuerdo con el hecho de que contar con la infraestructura necesaria es un factor favorecedor de la inclusión del aprendizaje móvil en la educación superior; el mismo valor le dieron a la capacitación y a la formación docente en el uso y aplicación de los dispositivos móviles; esto seguido de un $80 \%$ del personal docente en cuanto a su interés por transformar su práctica académica y de un $63 \%$ por la gestión administrativa y las políticas IES.

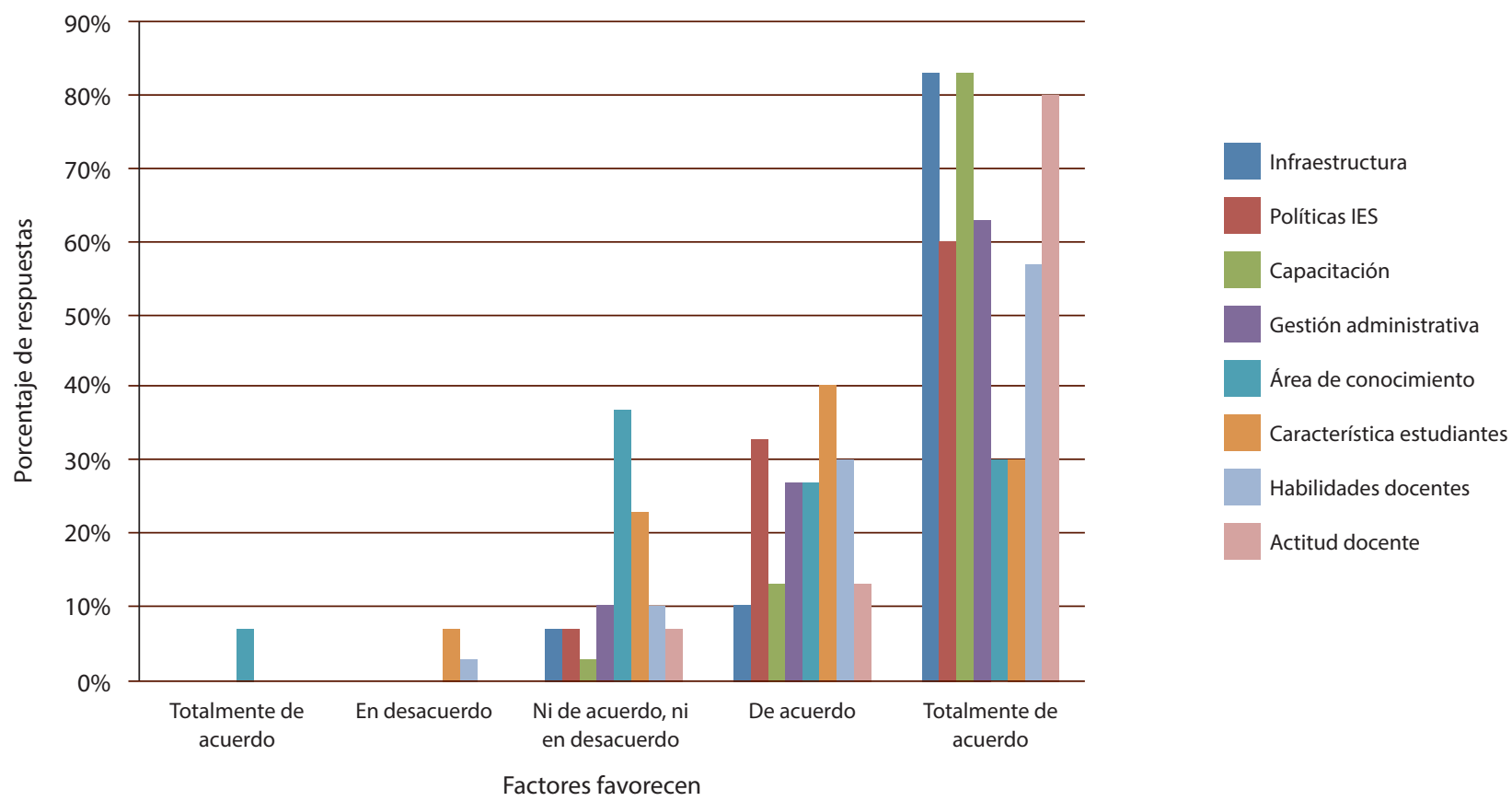

Figura 1: Porcentaje de respuestas para los factores que favorecen la inclusión del aprendizaje móvil. Nota: Tomado de Estrada-Villa (2014, p. 100).

Esto podría verse reflejado en que a la hora de integrar tecnología no es solo necesario pensar en la infraestructura ni en los recursos destinados para la capacitación, sino que además es indispensable la actitud y el interés por parte del personal docente para integrar los dispositivos móviles a su práctica educativa. Aunque el $30 \%$ del equipo investigador está totalmente de acuerdo con que el área de conocimiento influye a la hora de integrar, un $7 \%$ está en desacuerdo y para el $37 \%$ restante es irrelevante la disciplina a la hora de integrar 
doi: http://dx.doi.org/10.15359/ree.22-3.6

URL: http://www.una.ac.cr/educare

CORREO: educare@una.cr

tecnología. Adicionalmente, se evidenció que los dispositivos móviles son ideales para prácticas de medicina, aprendizaje de una segunda lengua y laboratorios de ciencias exactas, lo que no sucede en otros campos del conocimiento, como la filosofía o el derecho.

\section{Primer análisis: ACP para factores que favorecen el aprendizaje móvil}

Para analizar los hallazgos, se toman como variables los ocho factores que favorecen y que están consignados en la Tabla 2, que a su vez son fuente de la Figura 1.

Tabla 2: Respuestas de la encuesta "Factores Az Móvil" para los factores que favorecen la inclusión del m-learning en educación superior

\begin{tabular}{ccccccccc}
\hline Investigador & Factor 1 & Factor 2 & Factor 3 & Factor 4 & Factor 5 & Factor 6 & Factor 7 & Factor 8 \\
\hline Sujeto 1 & 5 & 5 & 5 & 5 & 5 & 5 & 5 & 5 \\
Sujeto 2 & 5 & 5 & 5 & 5 & 4 & 4 & 5 & 3 \\
Sujeto 3 & 5 & 5 & 5 & 5 & 4 & 5 & 5 & 5 \\
Sujeto 4 & 3 & 3 & 4 & 3 & 3 & 3 & 3 & 3 \\
Sujeto 5 & 5 & 5 & 5 & 5 & 4 & 3 & 5 & 3 \\
Sujeto 6 & 5 & 5 & 5 & 5 & 5 & 5 & 5 & 5 \\
Sujeto 7 & 5 & 4 & 5 & 5 & 3 & 5 & 4 & 5 \\
Sujeto 8 & 5 & 5 & 5 & 5 & 4 & 5 & 4 & 4 \\
Sujeto 9 & 5 & 5 & 5 & 5 & 3 & 5 & 5 & 3 \\
Sujeto 10 & 5 & 5 & 5 & 5 & 4 & 5 & 5 & 3 \\
Sujeto 11 & 5 & 5 & 5 & 5 & 5 & 5 & 5 & 4 \\
Sujeto 12 & 5 & 5 & 5 & 5 & 4 & 5 & 5 & 1 \\
Sujeto 13 & 5 & 5 & 5 & 5 & 4 & 5 & 5 & 4 \\
Sujeto 14 & 4 & 5 & 4 & 5 & 2 & 5 & 5 & 5 \\
Sujeto 15 & 5 & 5 & 3 & 5 & 3 & 4 & 5 & 4 \\
Sujeto 16 & 4 & 4 & 4 & 4 & 3 & 2 & 4 & 4 \\
Sujeto 17 & 5 & 5 & 4 & 5 & 5 & 5 & 5 & 4 \\
Sujeto 18 & 5 & 5 & 5 & 5 & 5 & 5 & 5 & 5 \\
& & & & & & & & continúa
\end{tabular}


doi: http://dx.doi.org/10.15359/ree.22-3.6

URL: http://www.una.ac.cr/educare

CORREO: educare@una.cr

\begin{tabular}{ccccccccc}
\hline Investigador & Factor 1 & Factor 2 & Factor 3 & Factor 4 & Factor 5 & Factor 6 & Factor 7 & Factor 8 \\
\hline Sujeto 19 & 5 & 5 & 5 & 5 & 4 & 4 & 5 & 4 \\
Sujeto 20 & 5 & 5 & 4 & 5 & 3 & 4 & 5 & 3 \\
Sujeto 21 & 5 & 5 & 4 & 5 & 4 & 4 & 4 & 5 \\
Sujeto 22 & 5 & 5 & 3 & 5 & 4 & 4 & 3 & 3 \\
Sujeto 23 & 5 & 4 & 4 & 5 & 5 & 4 & 5 & 5 \\
Sujeto 24 & 4 & 4 & 4 & 4 & 3 & 5 & 4 & 3 \\
Sujeto 25 & 5 & 5 & 5 & 5 & 4 & 3 & 4 & 4 \\
Sujeto 26 & 5 & 5 & 5 & 5 & 5 & 5 & 5 & 5 \\
Sujeto 27 & 5 & 5 & 5 & 3 & 4 & 4 & 4 & 3 \\
Sujeto 28 & 5 & 5 & 4 & 5 & 5 & 4 & 4 & 3 \\
Sujeto 29 & 5 & 5 & 4 & 4 & 5 & 5 & 5 & 3 \\
Sujeto 30 & 3 & 5 & 5 & 4 & 2 & 5 & 3 & 1 \\
\hline
\end{tabular}

* Las respuestas consignadas en la tabla se encuentran en valores de uno a cinco, uno es el valor más bajo y cinco el más alto 5= Totalmente de acuerdo, 4= De acuerdo, 3= Ni de acuerdo, ni en desacuerdo 2= En desacuerdo, 1= Totalmente en desacuerdo

Nota: Tomado de Estrada-Villa (2014, p. 104).

Tabla 3: Análisis de componentes principales factores favorecen

\begin{tabular}{lccc}
\hline \multicolumn{1}{c}{$\begin{array}{c}\text { Componentes } \\
\text { número }\end{array}$} & $\begin{array}{c}\text { Eigenvalue } \\
\text { (confianza) }\end{array}$ & $\begin{array}{c}\text { Porcentaje de } \\
\text { varianza (\%) }\end{array}$ & $\begin{array}{c}\text { Porcentajea } \\
\text { cumulado (\%) }\end{array}$ \\
\hline 1. Factor infraestructura & 3,44199 & 43,025 & 43,025 \\
2. Factor capacitación y formación docente & 1,25985 & 15,748 & 58,773 \\
3. Factor políticas IES & 0,914783 & 11,435 & 70,208 \\
4. Factor actitud e interés docente & 0,804202 & 10,053 & 80,260 \\
5. Factor características del estudiantado & 0,618168 & 7,727 & 87,987 \\
6. Factor conocimiento y habilidades docentes & 0,453355 & 5,667 & 93,654 \\
7. Factor gestión administrativa & 0,307412 & 3,843 & 97,497 \\
8. Factor área de conocimiento y campo de estudio & 0,200244 & 2,503 & 100,000 \\
\hline
\end{tabular}

Nota: Tomado de Estrada-Villa (2014, p.105). 
doi: http://dx.doi.org/10.15359/ree.22-3.6

URL: http://www.una.ac.cr/educare

CORREO: educare@una.cr

Variabilidad: $58,7729 \%$

Número de factores extraídos: 2

Entrada de datos: observaciones

Número de casos completos: 30

Tratamiento de valores perdidos: eliminación listwise

Tipo de factorización: componentes principales

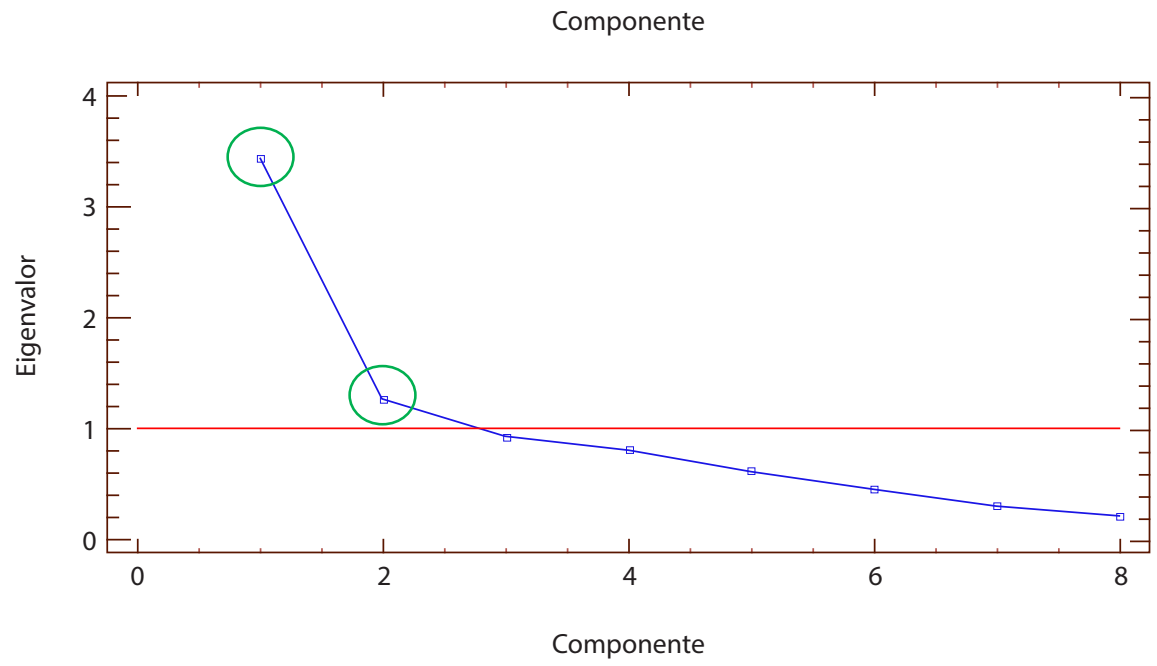

Figura 2: Diagrama de los factores (componentes) que favorecen extraídos

Nota: Tomado de Estrada-Villa (2014, p. 105).

El análisis muestra que dos componentes se han extraído, puesto que dos factores tuvieron autovalores mayores o iguales que 1,0 (ver Figura 2). En conjunto ellos se explican con un $58,7729 \%$ de la variabilidad en los datos originales. Desde el valor de la confianza, en la Tabla 3 se observa que los valores mayores que uno se encuentran en los componentes 1 y 2; por tanto, los factores arrojados por el ACP son: factor infraestructura, con 3,44199, y factor capacitación y formación docente, con 1,25985.

\section{Segundo análisis: ANOVA multifactorial para factores que favorecen el aprendizaje móvil}

El ANOVA es un análisis de varianza de diversas variables dependientes en relación con la variable independiente. En este caso se utiliza para determinar cuáles de los factores que favorecen el m-learning en educación superior tienen un efecto estadísticamente significativo 
sobre la variable dependiente; asimismo, sirve para evaluar la significancia de las interacciones entre los mencionados factores.

Para realizar el análisis se seleccionó por la varianza la variable dependiente; en este caso los datos arrojaron que es el factor capacitación y formación docente versus /as variables independientes: factor infraestructura, factor políticas IES, factor actitud e interés docente, factor características del estudiantado, factor conocimiento y habilidades docentes, factor gestión administrativa, factor área de conocimiento y campo de estudio.

Tabla 4: Análisis de varianza para el factor capacitación y formación docente como factor que favorece el m-learning y suma de cuadrados tipo III

\begin{tabular}{llllll}
\hline \multicolumn{1}{c}{ Efectos principales } & $\begin{array}{c}\text { Suma de } \\
\text { cuadrados }\end{array}$ & Gl & $\begin{array}{c}\text { Cuadrado } \\
\text { medio }\end{array}$ & Razón-F & Valor-P \\
\hline A:_infraestructura & 0,351886 & 2 & 0,175943 & 1,82 & 0,2047 \\
B:__políticas de las IES y lineamientos & 0,149592 & 2 & 0,0747962 & 0,77 & 0,4838 \\
C:_actitud e interés del docente & 0,0466716 & 2 & 0,0233358 & 0,24 & 0,7897 \\
D:__características del estudiantado & 1,6059 & 3 & 0,535301 & 5,52 & 0,0129 \\
E:__conocimiento y habilidades de docentes & 0,0609446 & 3 & 0,0203149 & 0,21 & 0,8878 \\
F:__gestión administrativa & 0,0855064 & 2 & 0,0427532 & 0,44 & 0,6533 \\
G:_área de conocimiento y campo de & 0,350526 & 3 & 0,116842 & 1,21 & 0,3496 \\
estudio & & & & & \\
Residuos & 1,16276 & 12 & 0,096897 & & \\
\hline Total (corregido) & 6,8 & 29 & & & \\
\hline
\end{tabular}

*Todas las razones-F se basan en el cuadrado medio del error residual Número de casos completos: 30

Nota: Elaboración propia con el apoyo del programa Statgraphics.

El análisis de la varianza determinó cuáles de los factores que favorecen el $m$-Learning en la educación superior tienen un efecto estadístico significativo sobre el factor capacitación y formación docente. Por tanto, el ANOVA (ver Tabla 4) descompone la variabilidad del factor capacitación docente en contribuciones debidas a varios factores, puesto que se ha escogido la suma de cuadrados tipo III (por omisión del software estadístico statgraphics). La contribución de cada factor se mide eliminando los efectos de los demás. Los valores-P prueban la significancia estadística de cada uno de los factores, ya que un valor-P es menor que 0,05 
doi: http://dx.doi.org/10.15359/ree.22-3.6

URL: http://www.una.ac.cr/educare

CORREO: educare@una.cr

y corresponde al factor características de estudiantes 0,0129; por tanto, este factor tiene un efecto estadísticamente significativo sobre el factor capacitación y formación docente, con un $95 \%$ de nivel de confianza.

En suma, los factores capacitación docente y factor características de estudiantes, extraídas de los respectivos análisis, constituyen una herramienta de pronóstico. Esto significa que, si se intervienen las variables capacitación docente y características de estudiantes, es decir, desarrollando una serie de actividades que fortalezcan la capacitación en el uso del $m$-learning que tenga como base las actividades que está demandando el estudiantado en el aula, habría una probabilidad de un $95 \%$ de favorecer la inclusión del aprendizaje móvil.

\section{Análisis multifactorial para los factores que dificultan el m-learning en educación superior en Colombia}

Por otro lado, y continuando con el análisis, el grupo investigador afirma que las dificultades para la inclusión del aprendizaje móvil en educación superior (ver Figura 3) se presentan por el desconocimiento (63\%) de las posibilidades de los dispositivos móviles, por la actitud y el desinterés docente (63\%), así como por la falta de capacitación (50\%) a docentes. La ausencia de infraestructura (43\%) de conectividad y acceso a internet es un factor determinante, así como la no existencia de gestión administrativa (53\%) y de lineamientos (53\%) a nivel nacional e institucional que incentive su uso en los procesos de enseñanza aprendizaje.

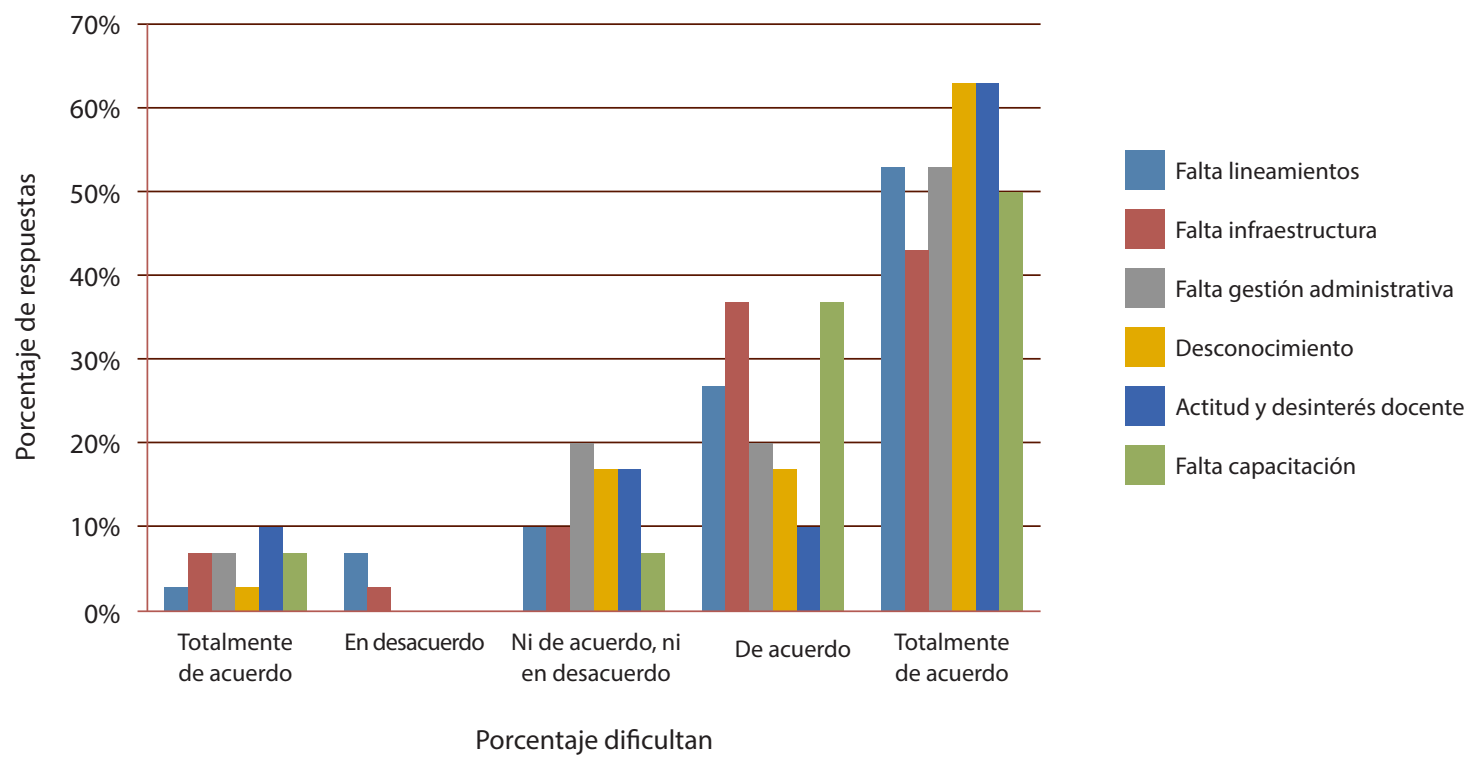

Figura 3: Porcentaje de respuestas para los factores que dificultan Nota:Tomado de Estrada-Villa (2014, p. 102). 


\section{Primer análisis: ACP para factores que dificultan el aprendizaje móvil}

Este procedimiento ejecuta un análisis de componentes principales. Como se mencionó anteriormente, el propósito del ACP es obtener un número reducido de las seis variables que expliquen la mayor variabilidad en los datos. Los datos fuente están consignados en la Tabla 5.

Tabla 5: Resultado de la encuesta realizado al grupo experto en educación a partir del muestreo bola de nieve para los factores que dificultan la inclusión del m-learning en la educación superior

\begin{tabular}{|c|c|c|c|c|c|c|}
\hline $\begin{array}{l}\text { Investigador/ } \\
\text { Investigadora }\end{array}$ & $\begin{array}{c}\text { Falta gestión } \\
\text { administrativa }\end{array}$ & $\begin{array}{c}\text { Falta } \\
\text { infraestructura }\end{array}$ & $\begin{array}{c}\text { Falta lineamientos } \\
\text { m-learning }\end{array}$ & Desconocimiento & $\begin{array}{c}\text { Falta } \\
\text { capacitación }\end{array}$ & $\begin{array}{c}\text { Actitud y desinterés } \\
\text { docente }\end{array}$ \\
\hline Sujeto 1 & 1 & 1 & 2 & 4 & 3 & 1 \\
\hline Sujeto 2 & 4 & 4 & 4 & 5 & 4 & 5 \\
\hline Sujeto 3 & 5 & 5 & 5 & 5 & 5 & 5 \\
\hline Sujeto 4 & 3 & 3 & 3 & 3 & 3 & 3 \\
\hline Sujeto 5 & 5 & 5 & 5 & 3 & 3 & 5 \\
\hline Sujeto 6 & 5 & 5 & 5 & 5 & 5 & 5 \\
\hline Sujeto 7 & 4 & 5 & 2 & 5 & 5 & 5 \\
\hline Sujeto 8 & 1 & 1 & 2 & 4 & 1 & 1 \\
\hline Sujeto 9 & 5 & 4 & 5 & 5 & 5 & 4 \\
\hline Sujeto 10 & 3 & 2 & 5 & 3 & 5 & 3 \\
\hline Sujeto 11 & 5 & 5 & 5 & 5 & 5 & 5 \\
\hline Sujeto 12 & 5 & 5 & 5 & 5 & 4 & 5 \\
\hline Sujeto 13 & 5 & 4 & 5 & 5 & 5 & 5 \\
\hline Sujeto 14 & 5 & 4 & 5 & 4 & 4 & 1 \\
\hline Sujeto 15 & 5 & 4 & 5 & 5 & 5 & 5 \\
\hline Sujeto 16 & 4 & 4 & 4 & 3 & 4 & 3 \\
\hline Sujeto 17 & 5 & 5 & 5 & 5 & 5 & 5 \\
\hline Sujeto 18 & 5 & 5 & 5 & 5 & 5 & 5 \\
\hline Sujeto 19 & 5 & 5 & 5 & 5 & 5 & 5 \\
\hline Sujeto 20 & 5 & 4 & 4 & 5 & 4 & 5 \\
\hline Sujeto 21 & 3 & 5 & 3 & 3 & 4 & 3 \\
\hline Sujeto 22 & 3 & 4 & 3 & 5 & 5 & 5 \\
\hline Sujeto 23 & 3 & 5 & 4 & 4 & 4 & 4 \\
\hline Sujeto 24 & 4 & 4 & 4 & 5 & 4 & 3 \\
\hline Sujeto 25 & 4 & 4 & 4 & 4 & 4 & 4 \\
\hline Sujeto 26 & 5 & 5 & 5 & 5 & 5 & 5 \\
\hline Sujeto 27 & 5 & 4 & 5 & 4 & 4 & 5 \\
\hline Sujeto 28 & 5 & 5 & 4 & 5 & 4 & 5 \\
\hline Sujeto 29 & 4 & 3 & 4 & 5 & 5 & 5 \\
\hline Sujeto 30 & 3 & 3 & 5 & 5 & 5 & 5 \\
\hline
\end{tabular}

* Las respuestas consignadas en la tabla se encuentra en valores de uno a cinco, uno es el valor más bajo y cinco el más alto $5=$ Totalmente de acuerdo, 4= De acuerdo, 3= Ni de acuerdo, ni en desacuerdo, 2= En desacuerdo, 1= Totalmente en desacuerdo. Nota:Tomado de Estrada-Villa (2014, p.107). 
doi: http://dx.doi.org/10.15359/ree.22-3.6

URL: http://www.una.ac.cr/educare

CORREO: educare@una.cr

Tabla 6: Análisis de componentes principales de los factores dificultan el m-learning

\begin{tabular}{lccc}
\hline \multicolumn{1}{c}{$\begin{array}{c}\text { Componentes } \\
\text { Número }\end{array}$} & $\begin{array}{c}\text { Eigenvalue } \\
\text { (confianza) }\end{array}$ & $\begin{array}{c}\text { Porcentaje de } \\
\text { varianza (\%) }\end{array}$ & $\begin{array}{c}\text { Porcentaje } \\
\text { acumulado (\%) }\end{array}$ \\
\hline 1. Falta gestión administrativa & 3,76256 & 62,709 & 62,709 \\
2. Falta infraestructura & 0,871654 & 14,528 & 77,237 \\
3. Falta lineamientos m-learning & 0,579405 & 9,657 & 86,894 \\
4. Desconocimiento & 0,401688 & 6,695 & 93,589 \\
5. Falta capacitación & 0,278796 & 4,647 & 98,235 \\
6. Actitud y desinterés & 0,105894 & 1,765 & 100,000 \\
\hline
\end{tabular}

Nota:Tomado de Estrada-Villa (2014, p. 108).

Variabilidad: 62,709

Número de componentes extraídos: 1

Entrada de datos: observaciones

Número de casos completos: 30

Tratamiento de valores perdidos: eliminación listwise

Tipo de factorización: componentes principales

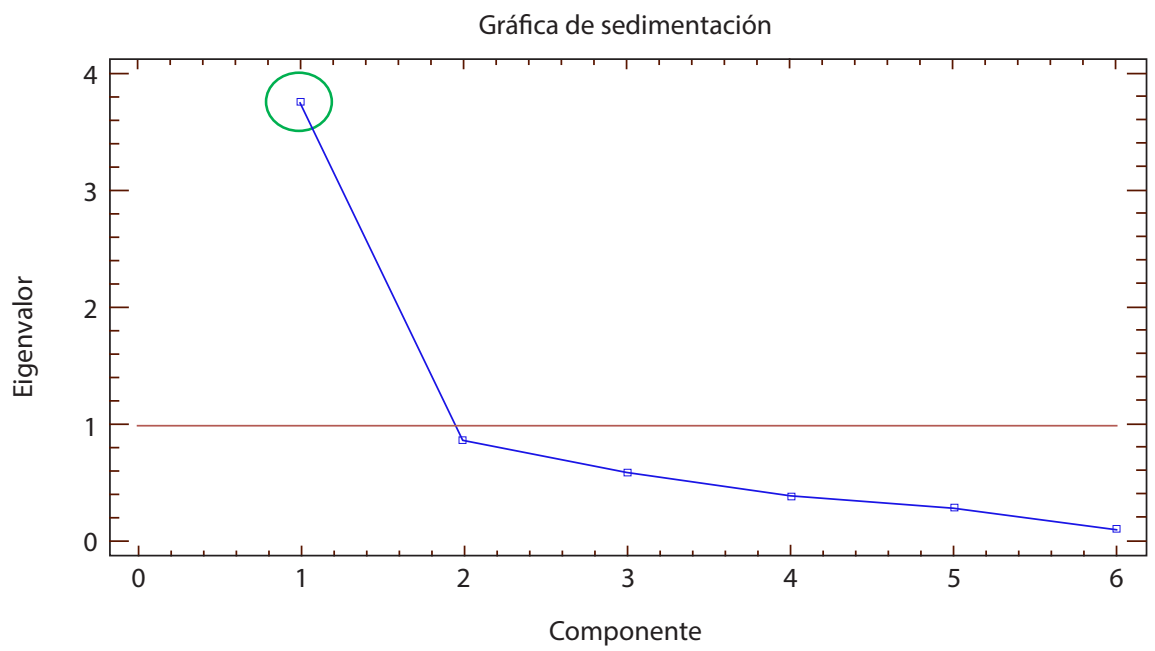

Figura 4: Diagrama del análisis de componentes principales para los factores que dificultan.

Nota: Tomado de Estrada-Villa (2014, p. 107).

En este caso, un componente ha sido extraído (ver Figura 4), puesto que es el único componente con un autovalor mayor o igual que 1,0, que corresponde al factor falta de gestión administrativa, con un valor de 3,76256 (ver Tabla 6). El conjunto de componentes se explica con un $62,7094 \%$ de la variabilidad en los datos originales. 


\section{Segundo análisis: ANOVA multifactorial para los factores que dificultan el aprendizaje móvil en educación superior}

Se realizó el análisis de varianza de cinco factores relacionados con el factor falta de gestión administrativa, con el fin de determinar cuáles de los factores que dificultan el m-learning en educación superior tienen un efecto estadísticamente significativo sobrelavariableindependiente.

La variable independiente se seleccionó a partir del factor con la varianza más alta, que en este caso es la falta de gestión administrativa, y se contrastó en el ANOVA frente a las variables independientes: falta infraestructura, falta lineamientos m-learning, desconocimiento docente, falta de capacitación, actitud y desinterés docente.

Tabla 7: Análisis de varianza para el factor falta de gestión administrativa como factor que dificulta el m-Learning y suma de cuadrados tipo III

\begin{tabular}{lccccc}
\hline Efectos principales & Suma de cuadrados & $\mathrm{Gl}$ & Cuadrado medio & Razón-F & Valor-P \\
\hline A: Falta infraestructura & 5,28186 & 4 & 1,32047 & 5,42 & 0,0075 \\
B: Falta lineamientos m-learning & 4,22993 & 3 & 1,40998 & 5,79 & 0,0087 \\
C: Desconocimiento & 0,248089 & 2 & 0,124045 & 0,51 & 0,6117 \\
D: Falta capacitación & 0,392795 & 3 & 0,130932 & 0,54 & 0,6642 \\
E: Actitud y desinterés & 0,509317 & 3 & 0,169772 & 0,70 & 0,5692 \\
Residuos & 3,41011 & 14 & 0,24358 & & \\
\hline Total (corregido) & 39,4667 & 29 & & & \\
\hline
\end{tabular}

*Todas las razones-F se basan en e I cuadrado medio del error residual Número de casos completos: 30

Nota: Elaboración propia con el apoyo de statgraphics.

Las pruebas-F en la tabla ANOVA (ver Tabla 7) permitieron identificar los factores significativos. Para estos últimos, las pruebas de rangos múltiples evidencian cuáles medias son significativamente diferentes de otras.

La Tabla 7 descompone la variabilidad del factor falta de gestión administrativa en contribuciones debidas a varios factores. Los valores-P prueban la significancia estadística de cada uno de los factores. Puesto que dos valores-P son menores que 0,05 , que son: factor falta de infraestructura, con un valor-P de 0,0075 y falta de lineamientos m-learning, con un valor-P de 0,0087 . Estos factores tienen un efecto estadísticamente significativo sobre la variable dependiente del análisis, con un $95,0 \%$ de nivel de confianza. 
doi: http://dx.doi.org/10.15359/ree.22-3.6

URL: http://www.una.ac.cr/educare

CORREO: educare@una.cr

A partir de estos resultados, se deduce que sí se controlan las variables falta de infraestructura y la falta de políticas IES, con una serie de actividades enfocadas al fortalecimiento de la infraestructura por parte de la administración de una IES, se podrá obtener un 95\% de éxito en la integración de los dispositivos moviles.

\section{Sintesis de resultados}

A partir de los análisis realizados se infiere que el factor capacitación docente depende directamente del factor características del estudiantado. Estos factores, al estar correlacionados, son los que podrian facilitar la integración del aprendizaje móvil en las actividades académicas en educación superior. Por tanto, el factor capacitación docente es explicado en un $95 \%$ por la variable características del estudiantado (ver Tabla 8). En concordancia, la formación docente constituye un factor que contribuye a la inclusión del aprendizaje móvil en educación superior.

Tabla 8: Resumen de hallazgos por análisis desarrollado para los factores que facilitan la inclusión del aprendizaje móvil en educación superior

\begin{tabular}{|c|c|c|}
\hline Instrumento & Desarrollo del análisis & Resultados de los análisis \\
\hline \multirow{10}{*}{ 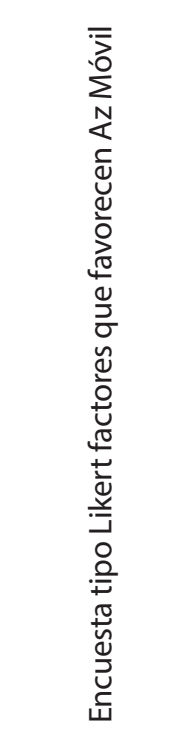 } & \multirow{5}{*}{$\begin{array}{l}\text { Primer análisis } \\
\text { Análisis de componentes principales } \\
\text { variables: infraestructura, capacitación, } \\
\text { políticas IES, actitud e interés docente, } \\
\text { conocimiento y habilidades, gestión } \\
\text { administrativa, área de conocimiento. }\end{array}$} & El ACP extrae los siguientes dos factores que \\
\hline & & favorecen el m-learning en educación superior: \\
\hline & & *Infraestructura con 3,44199 de Confianza \\
\hline & & *Capacitación con 1,25985 de confianza \\
\hline & & $\begin{array}{l}\text { En conjunto, los factores se extraen con un } \\
58,7729 \% \text { de variabilidad de los datos originales. }\end{array}$ \\
\hline & \multirow{5}{*}{$\begin{array}{l}\text { Segundo Análisis } \\
\text { ANOVA multifactorial } \\
\text { Variable dependiente } Y=\text { Capacitación y } \\
\text { formación docente } \\
\qquad \text { vs. } \\
\text { Variables independientes } X=\text { Infraestructura, po- } \\
\text { líticas IES, actitud e interés docente, característi- } \\
\text { cas de estudiantes, conocimiento y habilidades, } \\
\text { gestión administrativa, área de conocimiento. }\end{array}$} & El valor-P en el ANOVA prueba la significancia \\
\hline & & estadística de los factores que favorecen el \\
\hline & & m-learning, que en este caso son: \\
\hline & & $\begin{array}{l}\text { *Valor-P factor características de } \\
\text { estudiantes }=0,0129\end{array}$ \\
\hline & & $\begin{array}{l}\text { El factor anterior tiene un valor significativo } \\
\text { sobre el factor capacitación y formación docente } \\
\text { con un } 95 \% \text { de nivel de confianza. }\end{array}$ \\
\hline
\end{tabular}

Nota:Tomado de Estrada-Villa (2014, p. 116).

De la misma manera, se realizó el tratamiento de los datos para los factores que dificultan, donde los hallazgos mostraron que el factor falta de gestión administrativa depende de la falta de políticas IES y lineamientos m-learning y de la infraestructura, por tanto, estos factores son 
determinantes a la hora de llevar los dispositivos móviles al aula (ver Tabla 9). Por lo anterior, la infraestructura es un factor que dificulta la incluciòn del m-learning en educación superior.

Tabla 9: Resumen de hallazgos por análisis para los factores que dificultan la inclusión del aprendizaje móvil en educación superior

\begin{tabular}{|c|c|c|}
\hline Instrumento & Desarrollo del análisis & Resultados de los análisis \\
\hline \multirow{9}{*}{ 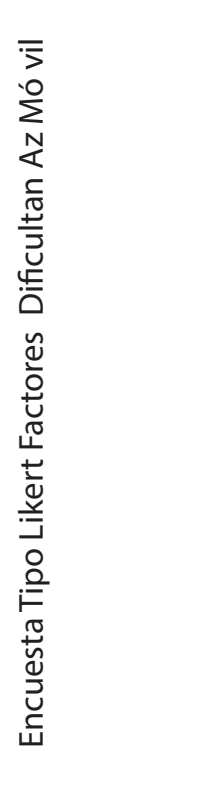 } & Primer análisis & \multirow{2}{*}{$\begin{array}{l}\text { El ACP reduce de seis a uno los factores que } \\
\text { dificultan el m-learning en educación superior: }\end{array}$} \\
\hline & Análisis de componentes principales & \\
\hline & $\begin{array}{l}\text { Variables: falta gestión administrativa, falta } \\
\text { infraestructura, falta lineamientos m-learning, } \\
\text { desconocimiento, falta de capacitación, } \\
\text { actitud y desinterés docente }\end{array}$ & $\begin{array}{l}\text { *Factor falta de gestión administrativa con } \\
\text { un valor de } 3,76256 \text { de confianza y con un } \\
\text { porcentaje de variabilidad del } 80,219 \% \text {. }\end{array}$ \\
\hline & Segundo Análisis & \multirow{3}{*}{$\begin{array}{l}\text { El valor-P en el ANOVA prueba la significancia } \\
\text { estadística de los factores que dificultan el } \\
\text { m-learning, que en este caso son: }\end{array}$} \\
\hline & Anova Multifactorial & \\
\hline & Variable Dependiente $Y=$ Falta de Gestión & \\
\hline & Administrativa & ${ }^{*}$ Valor-P falta de infraestructura $=0,0075$ \\
\hline & 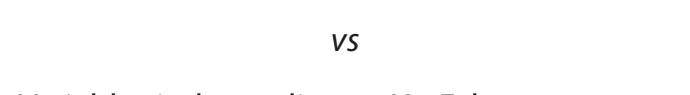 & $\begin{array}{l}\text { *Valor-P falta de lineamientos m-learning } \\
=0.0087\end{array}$ \\
\hline & $\begin{array}{l}\text { Variables independientes } X=\text { Falta } \\
\text { Infraestructura, Falta Políticas IES y } \\
\text { Lineamientos mlearning, Desconocimiento, } \\
\text { Falta de Capacitación, Actitud y Desinteres } \\
\text { Docente }\end{array}$ & $\begin{array}{l}\text { Los factores anteriores tienen un valor } \\
\text { significativo sobre el factor falta de gestión } \\
\text { administrativa con un } 95 \% \text { de nivel de } \\
\text { confianza. }\end{array}$ \\
\hline
\end{tabular}

Nota:Tomado de Estrada-Villa (2014, p. 116).

\section{Conclusiones}

Los dispositivos móviles, por su portabilidad yentornos ricos en interacción y comunicación, acercan y permean la acción formativa, y de esta manera trascienden el aula de clase y generan escenarios personalizados (Bruce, 2008) y ajustados a las necesidades del estudiantado; además, validan los estilos de aprendizaje, con lo cual aportan al aprendizaje colaborativo.

Esto, puesto que, como lo menciona Attewell (2005), los teléfonos móviles ya no son solo para chatear y organizar contactos o la agenda, pues se han convertido en computadoras de bolsillo y, por tanto, tienen la capacidad de entregar objetos de aprendizaje y proporcionar acceso a sistemas y servicios.

Ahora bien, la inclusión del m-learning da la posibilidad a la comunidad académica de acceder a la información desde cualquier lugar, permite la interacción con otro estudiantado y acceder a fuentes de información digitales, es por esto que se proyecta como el futuro de la educación aplicable en muchas áreas. 
doi: http://dx.doi.org/10.15359/ree.22-3.6

URL: http://www.una.ac.cr/educare

CORREO: educare@una.cr

Los hallazgos plantean retos para la gestión administrativa de las IES e invitan al profesorado universitario a reflexionar sobre su práctica docente, dadas las posibilidades actuales de incluir los dispositivos móviles a las actividades académicas.

Asimismo, los resultados arrojan que se deben delimitar unas políticas que trasciendan al aula (Organización para la Cooperación y el Desarrollo Económicos [OCDE], Banco Internacional de Reconstrucción y Fomento, y Banco Mundial, 2013) y que orienten la vinculación del $m$-learning en la práctica educativa.

Por esto, uno de los aspectos que cabe resaltar a partir de los resultados encontrados es que tanto las universidades, como los cuerpos docentes deben estar abiertos y preparados al cambio, en cuanto lo relacionado con la actualización de la infraestructura, así como con la capacitación académica en torno a la integración del aprendizaje móvil. En definitiva, los factores identificados deben ser tenidos en cuenta a la hora de incluir el aprendizaje móvil, por tanto, que las universidades colombianas deben tomar la información base y aprovechar los factores aquí extraídos, con el fin de trazar políticas y lineamientos para incluir el aprendizaje móvil en la educación superior. Estas, a su vez, tienen que estar alineadas con la gestión administrativa desde planes estratégicos o de desarrollo, para que dichos factores puedan descender a través de planes de formación docente, y que se vea reflejada, en la práctica educativa, la incorporación de los dispositivos móviles, en respuesta a lo que el estudiantado está demandando.

Por ultimo, el m-learning, como una buena parte de lo relacionado con la inclusión de la tecnología en la educación y en otras áreas de la vida, sigue siendo un asunto en desarrollo, por lo cual mucho de lo que se dice o hace realmente forma parte de una exploración permanente por parte del personal docente, el estudiantado y las instituciones.

\section{Referencias}

Attewell, J. (2005). Mobile technologies and learning: A technology update and m-learning project summary. London: Learning and Skills Development Agency. Recuperado de http://wcc. wilkescc.edu/DeborahMcGuire/website/camtasiatutorials/CIT/Flickr/Mobile\%20Learning.pdf

Bernal, C.A (2000). Metodología de la investigación para administración y economía. Bogotá: Pearson.

Bruce, B. (2008). Ubiquitous learning, ubiquitous computing, and lived experience. En B. Cope y M. Kalantzis (Eds.), Ubiquitous learning (pp. 21-30). Champaign: University of Illinois Press. Recuperado de http://www.elizabethdelacruz.com/uploads/5/4/3/6/5436943/ ubiquitouslearning28nov07.pdf 
Estrada-Villa, E. J. (2014). Factores que contribuyen y dificultan el desarrollo de la enseñanza aprendizaje mediada por dispositivos móviles en instituciones de educación superior en Colombia (Tesis de maestría). Universidad de La Sabana, Chía, Colombia. Recuperado de $\quad$ https://intellectum.unisabana.edu.co/bitstream/handle/10818/11596/Erika\%20 Juliana\%20Estrada\%20Villa\%20\%28tesis\%29.pdf?sequence=1\&isAllowed=y

Estrada-Villa, E. J. (2016). Código QR basado en el aprendizaje móvil como estrategia para la investigación formativa: Un caso piloto. Ciencia y Poder Aéreo, 11(1), 230-241. doi: https:// doi.org/10.18667/cienciaypoderaereo.500

Hernández, R., Fernández, C. y Baptista, P. (2010). Metodología de la investigación (5ª ed.). México: McGraw-Hill.

Johnson, L., Adams, S. y Cummins, M. (2012). Informe horizon del NMC: Edición para la enseñanza universitaria 2012. Austin, Tejas: The New Media Consortium. Recuperado de http:// puntodeencuentro.utn.edu.ar/wp-content/uploads/2010/12/2012-horizon-report-HEspanish.pdf

O'Malley, C., Vavoula, G., Glew, J., Taylor, J., Sharples, M., Lefrere, P., ... Waycott, J. (2005). Guidelines for learning/teaching/tutoring/in a mobile environment. Mobilearn project (D.r.1), 1-84. Recuperado de https://hal.archives-ouvertes.fr/hal-00696244/document

Organización para la Cooperación y el Desarrollo Económicos, Banco Internacional de Reconstrucción y Fomento y Banco Mundial. (2013). Evaluaciones de políticas nacionales de educación: La educación superior en Colombia. Paris: OECD. doi: https://doi. org/10.1787/9789264180710-es

Sánchez, J., Sáenz, M., Muñoz, M., Ramírez, G. y Martín, S. (2009). Situación actual del m-learning. Recuperado de http://remo.det.uvigo.es/solite/images/pdf/situacin\%20actual\%20 del\%20m-learning\%20solite.pdf

Terrádez, M. (s. f.). Análisis de componentes principales. Recuperado de https://www.uoc.edu/ in 3/emath/docs/Componentes principales.pdf

Unesco. (2012). Mobile learning and policies. Key issues to consider. Paris: Autor. Recuperado de http://unesdoc.unesco.org/images/0021/002176/217638E.pdf 